\title{
Mapping the Distributions of Exoplanet Populations with NICI and GPI
}

\author{
Eric L. Nielsen ${ }^{1,2}$, Michael C. Liu ${ }^{3}$, Zahed Wahhaj ${ }^{4}$, Beth A. Biller ${ }^{5}$, \\ Thomas L. Hayward ${ }^{6}$, Laird M. Close ${ }^{7}$, the Gemini NICI \\ Planet-Finding Campaign Team,
} Bruce Macintosh ${ }^{2}$, Dmitry Savransky ${ }^{8}$, Jason J. Wang ${ }^{9}$, James R. Graham $^{9}$, Robert J. De Rosa ${ }^{9}$, Abhijith Rajan ${ }^{10}$, and the GPIES Consortium

\author{
${ }^{1}$ SETI Institute, 189 Bernardo Ave. Suite 100, Mountain View, CA 94043. email: \\ enielsen@seti.org ${ }^{2}$ Kavli Institute for Particle Astrophysics and Cosmology, 452 Lomita \\ Mall, Stanford, CA 94305. ${ }^{3}$ Institute for Astronomy, University of Hawaii at Manoa. \\ ${ }^{4}$ European Southern Observatory. ${ }^{5}$ Institute for Astronomy, University of Edinburgh. ${ }^{6}$ Gemini \\ Observatory. ${ }^{7}$ Steward Observatory, University of Arizona. ${ }^{8}$ Cornell University. ${ }^{9}$ University of \\ California, Berkeley. ${ }^{10}$ Arizona State University.
}

\begin{abstract}
While more and more long-period giant planets are discovered by direct imaging, the distribution of planets at these separations ( $\gtrsim 5 \mathrm{AU}$ ) has remained largely uncertain, especially compared to planets in the inner regions of solar systems probed by RV and transit techniques. The low frequency, the detection challenges, and heterogeneous samples make determining the mass and orbit distributions of directly imaged planets at the end of a survey difficult. By utilizing Monte Carlo methods that incorporate the age, distance, and spectral type of each target, we can use all stars in the survey, not just those with detected planets, to learn about the underlying population. We have produced upper limits and direct measurements of the frequency of these planets with the most recent generation of direct imaging surveys. The Gemini NICI Planet-Finding Campaign observed 220 young, nearby stars at a median H-band contrast of 14.5 magnitudes at 1", representing the largest, deepest search for exoplanets by the completion of the survey. The Gemini Planet Imager Exoplanet Survey is in the process of surveying 600 stars, pushing these contrasts to a few tenths of an arcsecond from the star. With the advent of large surveys (many hundreds of stars) using advanced planet-imagers we gain the ability to move beyond measuring the frequency of wide-separation giant planets and to simultaneously determine the distribution as a function of planet mass, semi-major axis, and stellar mass, and so directly test models of planet formation and evolution.
\end{abstract}

Keywords. instrumentation: adaptive optics, techniques: high angular resolution, planetary systems

\section{Introduction}

While over 1000 extrasolar planets have been found to date, only about a dozen have been directly imaged. Given the small angular separations $(\lesssim 1$ "), high contrasts between planets and stars $\left(10^{4}-10^{7}\right.$ for young giant planets), and low occurrence rate of intermediate-separation giant planets $(\lesssim 5 \%$, Nielsen \& Close 2010), large-scale surveys with advanced adaptive optics systems at large telescopes are required to find and characterize these planets. We have recently entered the era of multi-hundred star high contrast planet searches with surveys like NICI (Liu et al. 2010), IDPS (Vigan et al. 2012), SEEDS (Tamura et al. 2014), LEECH (Skemer et al. 2014), GPI (Macintosh et al. 2014a), and SPHERE (Beuzit et al. 2010). 

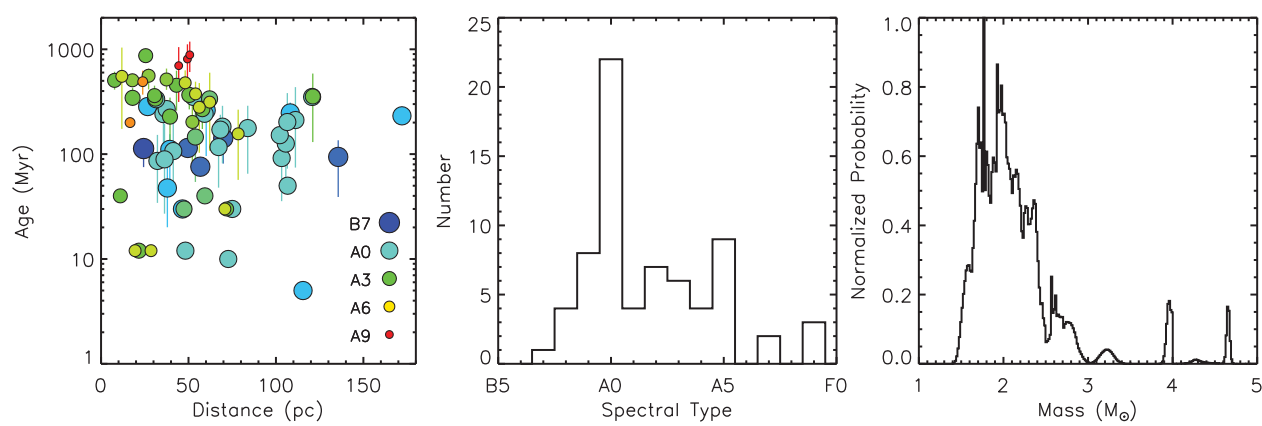

Figure 1. B and A stars observed by the Gemini NICI Planet-Finding Campaign. (Left) stars without error bars are members of known moving groups, while the rest have ages determined by our Bayesian method (Nielsen et al. 2013). (Center and right) spectral type and mass distributions of the B and A stars in the sample.

\section{The Gemini NICI Planet-Finding Campaign}

Using the Near Infrared Coronagraphic Imager at Gemini South, the Gemini NICI Planet-Finding Campaign surveyed 220 stars between 2008 and 2012 (Liu et al. 2010, Wahhaj et al. 2013a, Nielsen et al. 2013, Biller et al. 2013, Wahhaj et al. 2013b, Hayward et al. 2014). With median contrasts of 14.5 magnitude at 1", NICI represented the largest, deepest imaging search for planets conducted at the time. The target list was made up of a balance of spectral types from late to mid-M, with an emphasis on the youngest, nearest stars available in the southern sky. In addition to discovering 4 new brown dwarf companions (Biller et al. 2010, Wahhaj et al. 2011, Nielsen et al. 2012, Nielsen et al. 2013), the Campaign also charactered debris disks (Wahhaj et al. 2014, Biller et al. 2015) and the exoplanet $\beta$ Pic b (Males et al. 2014, Nielsen et al. 2014).

\subsection{The Ages of $B$ and $A$ stars}

Since the luminosity of giant planets is a strong function of age, determining ages is of key importance in carrying out direct imaging surveys, required to choose a target list, to properly characterize detected objects, and to evaluate survey completeness. B and A stars in particular are challenging, as the most common methods for evaluating age (e.g. calcium, lithium, X-ray, and rotation) aren't applicable for this range of mass. As part of the Gemini NICI Planet-Finding Campaign we developed a new Bayesian technique to produce accurate ages and uncertainties for these early-type stars given their position and errors in the color-magnitude diagrams, finding in many cases significantly older ages than have been previously reported in the literature (Nielsen et al. 2013). Figure 1 gives the derived ages and masses of our high-mass sample, as well as the distance and spectral type distribution.

\section{Completeness to Planets and Brown Dwarfs}

In order to evaluate survey completeness we utilize Monte Carlo simulations, as detailed in (Nielsen et al. 2005, 2008, and 2010). These simulations create an ensemble of simulated planets around each target star with full orbital parameters, and determine what fraction can be detected given the measured contrast curve for that star. By repeating over a grid of mass and semi-major axis we produce a completeness map for a single star, giving what fraction of planets, as a function of mass and orbital distance, can be detected with our observations. In the case of multiple epochs of contrast curves, the simulated planets are advanced forward in their orbits and compared to each contrast curve for that star. 


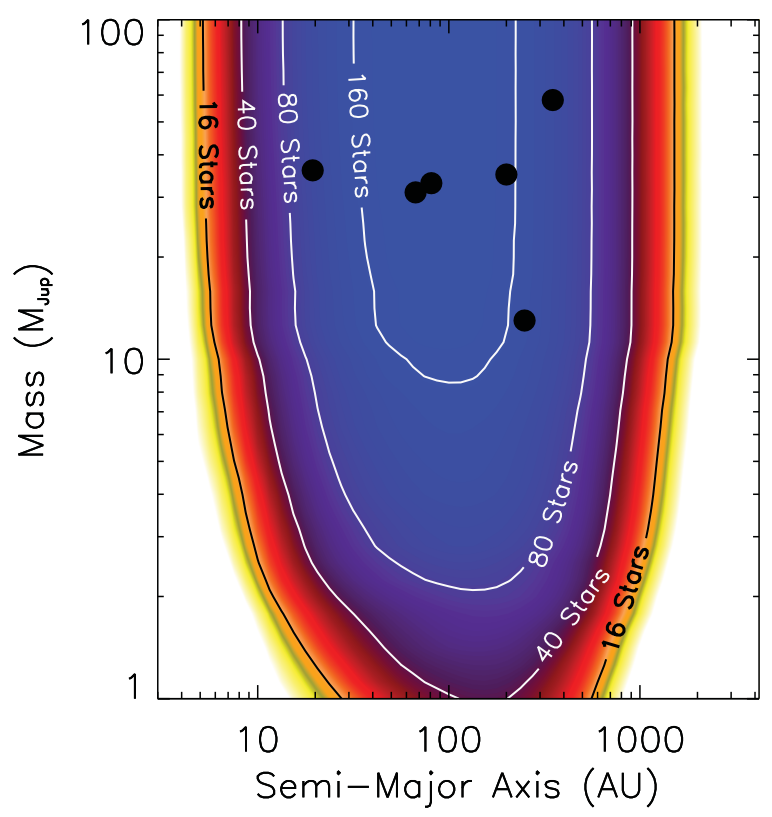

Figure 2. Completeness to planets and brown dwarfs from the NICI Campaign. Contours give the ranges of semi-major axis and companion mass for which the survey is complete to planets around the given number of stars. Black dots show detected brown dwarfs.

By summing these completeness maps for all stars we produce the completeness for the entire survey, as shown in Figure 2. The contours represent the number of stars for which we are $100 \%$ complete to planets of the given parameters (alternatively, if all stars had a planet of that mass and semi-major axis, the contour values give the number of planets we would expect to detect). Since the distances of target stars varied by over an order of magnitude, and no star is $100 \%$ complete to any type of planet (there are always some fraction of orbits that place the companion either behind or in front of the star, or off the detector), the survey completeness never reaches the total number of stars in the survey, 220. Nevertheless we show we were able to detect planets down to a few Jupiter masses at semi-major axes $\sim 20-800$ AU for a significant number of target stars.

By taking our survey completeness shown in Figure 2, expanding it into a fourdimensional completeness map to include stellar mass and projected separation, we can use a Bayesian method to compare the NICI-detected brown dwarfs to a model of brown dwarf populations. Using a series of power-law fits to brown dwarf mass, stellar host mass, and semi-major axis we find distributions consistent (at the $1 \sigma$ level) with similar fits to radial velocity giant planets presented by Cumming et al. (2008) and Johnson et al. (2010), despite the wide variation in mass and orbital separation between the two sets of populations. We do find that small-separation giant planets $(<2 \mathrm{AU})$ are 2-3 times more frequent than wide-separation (10-1000 AU) brown dwarfs, however.

\section{The Gemini Planet Imager Exoplanet Survey}

The Gemini Planet Imager (GPI) represents the latest technology for reaching high contrasts $(\sim 15$ magnitudes) close to the star $(<1 ")$, and so is able to image giant planets at the equivalent orbital distances of the giant planet region of our own solar system. The GPI Exoplanet Survey (GPIES) is currently searching for planets around 600 young, nearby stars (Macintosh et al. 2014a, 2014b, Perrin et al. 2014, 2015, Chilcote 


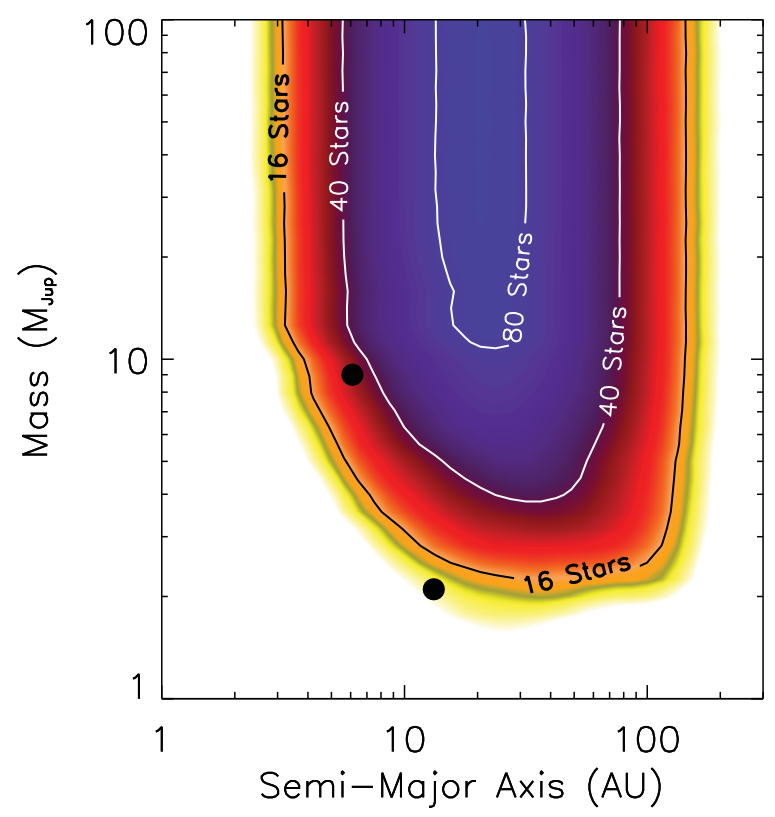

Figure 3. Completeness to planets and brown dwarfs from the first 93 stars of the GPIES Campaign. Note that the semi-major axis range of this plot has changed compared to Figure 2 to focus on the area of GPIES sensitivity, closer to the star compared to NICI.

et al. 2015). As of this writing, 93 stars have been observed by the GPIES campaign, and the first planet discovery is reported in Macintosh et al. (2015). We present the completeness to planets in Figure 3 for the GPIES Campaign. While the NICI instrument was able to reach deeper contrasts given its larger field of view (reaching contrasts of 17-18 magnitudes for some stars beyond 4"), GPI is probing smaller orbital distances compared to NICI. The new parameter space unseen by NICI but being studied by GPI runs from $\sim 5$ to $20 \mathrm{AU}$. Additionally, NICI's completeness reflects the fact that the NICI Campaign observed 220 stars compared to 93 for GPI, as the GPIES campaign continues the completeness will become deeper and move inward and outward in semi-major axis.

\section{Early Measurements of Exoplanet Populations}

In order to assess the constraints on planet populations we apply the same Bayesian technique we used for brown dwarf populations discussed in Section 3, this time for extrasolar giant planets. We combine the completeness from the NICI and GPIES results, as well as known and new planet detections. Our results are again broadly consistent with the RV results, finding similar power law distributions for 5-1000 AU giant planets as seen for $<2 \mathrm{AU}$ giant planets. Our frequency is consistent with that for RV planets as well, but may be slightly lower than that for the closer-in planets. Further observations and detections will allow us to make a more definitive comparison between these two sets of giant planet populations, and determine if they are in fact the same population, or if a break is evident that may trace evolution or formation mechanisms.

\section{Conclusions}

GPI's unprecedented sensitivity to small-separation giant planets is moving closer to the known radial velocity giant planets (Figure 4). At the current rate of observations 


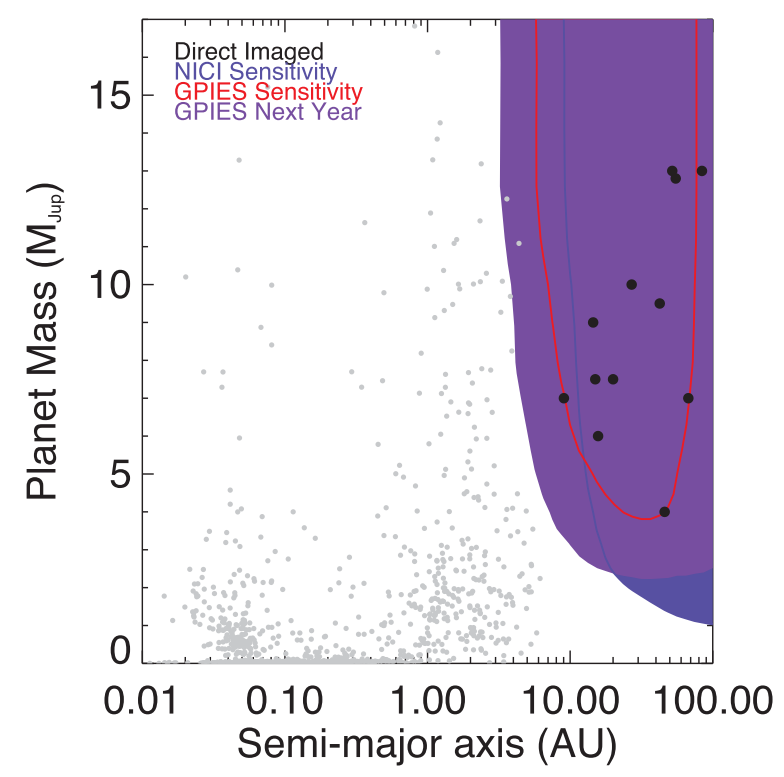

Figure 4. Known giant extrasolar planets from exoplanets.eu, and completeness from NICI and GPIES. Also shown is the projected completeness from the GPIES Campaign by June 2016, given the current rate of observations.

the number of stars observed by GPIES will rise from 93 to 300 in about a year from this writing, in June 2016. We can project out to that date by increasing the current completeness by a factor of three, which is the purple shaded region in Figure 4 . This region, corresponding to half of GPIES, overlaps known radial velocity giant planets, and suggests we will soon be closing the gap between RV and imaged planets. Within a year we can begin to measure giant exoplanets from the surface of the star out to thousands of $\mathrm{AU}$.

\section{References}

Beuzit, J. L., Boccaletti, A., Feldt, M., Dohlen, K., Mouillet, D., et al. 2010, ASPC, 430, 231. Biller, B. A., Liu, M. C., Wahhaj, Z., Nielsen, E. L., Close, L. M., et al. 2010, ApJ, 720, 82. Biller, B. A., Liu, M. C., Wahhaj, Z., Nielsen, E. L., Hayward, T. L., et al. 2013, ApJ, 777, 160. Biller, B. A., Liu, M. C., Rice, K., Wahhaj, Z., Nielsen, E. L., et al. 2015, MNRAS, 450, 4446. Cumming, A., Butler, R. P., March, G. W., Vogt, S. S., et al. 2008, PASP, 120, 531. Chilcote, J., Barman, T., Fitzgerald, M. P., Graham, J. R., et al. 2015, ApJ, 798, 3. Liu, M. C., Wahhaj, Z., Biller, B. A., Nielsen, E. L., Chun, M., et al. 2010, SPIE, 7736, 1. Hayward, T. L., Biller, B. A., Liu, M. C., Nielsen, E. L., et al. 2014, PASP, 126, 1112. Johnson, J. A., Aller, K. M., Howard, A. W., \& Crepp, J. R. 2010, PASP, 122, 905. Macintosh, B., Graham, J. R., Ingraham, P., Konopacky, Q., et al. 2014, PNAS, 11112661. Macintosh, B., Anthony, A., Atwood, J., Bauman, B., Cardwell, A., et al. 2014b, SPIE, 91480. Macintosh, B., Graham, J. R., Barman, T., De Rosa, R. J. et al. 2015, Science, in press. Males, J. R., Close, L. M., Morzinski, K. M., Wahhaj, Z., Liu, M. C., et al. 2014, ApJ, 786, 32. Nielsen, E. L., Close, L. M., Guirado, J. C., Biller, B. A., Lenzen, R., et al. 2005, AN, 326, 1033. Nielsen, E. L., Close, L. M., Biller, B. A., Masciadri, E., \& Lenzen, R. 2008, ApJ, 674, 466. Nielsen, E. L. \& Close, L. M. 2010, ApJ, 717, 878.

Nielsen, E. L., Liu, M. C., Wahhaj, Z., Biller, B. A., Hayward, T. L., et al. 2012, ApJ, 750, 53. Nielsen, E. L., Liu, M. C., Wahhaj, Z., Biller, B. A., Hayward, T. L., et al. 2013, ApJ, 776, 4. Nielsen, E. L., Liu, M. C., Wahhaj, Z., Biller, B. A., Hayward, T. L., et al. 2014, ApJ, 794, 158. 
Perrin, M. D., Maire, J., Ingraham, P., Savransky, D., et al. 2014, SPIE, 9147, 3.

Perrin, M. D., Duchene, G., Millar-Blanchaer, M., Fitzgerald, M. P., et al. 2015, ApJ, 799, 182.

Skemer, A. J., Hinz, P., Esposito, S., Skrutskie, M. F., Defrere, D., et al. 2014, SPIE, 9148, 0. Tamura, M., et al. 2014, IAUS, 299, 12.

Vigan, A., Patience, J., Marois, C., Bonavita, M., De Rosa, R. J., et al. 2012, A 8 A, 544, 9.

Wahhaj, Z., Liu, M. C., Biller, B. A., Clarke, F., Nielsen, E. L., et al. 2011, ApJ, 729, 139.

Wahhaj, Z., Liu, M. C., Nielsen, E. L., Biller, B. A., et al. 2013a, ApJ, 773, 179.

Wahhaj, Z., Liu, M. C., Biller, B. A., Nielsen, E. L., Hayward, T. L., et al. 2013b, ApJ, 779, 80.

Wahhaj, Z., Liu, M. C., Biller, B. A., Nielsen, E. L., Hayward, T. L., et al. 2014, ApJ, 567, 34.

\section{Discussion}

QUESTION: Does GPI really require astrometric follow-up given it's an IFS?

ANSWER: It's true that we have spectra for many of our candidate companions and thus have high confidence from a single observation that we're viewing background stars. However for completeness we follow up these objects with second epoch astrometric observations to confirm their nature as background objects as opposed to common proper motion companions. Unlike previous surveys with large fields of view (the NICI detector is 20 " $\mathrm{x} 20$ "), the GPI detector is 3 " on a side, so the number of background objects detected in our survey is small enough that getting second epochs is non prohibitive. Additionally, for the faintest candidates (corresponding to the lowest mass planets, if real) the candidates are seen only when all the wavelength channels are combined to increase S/N. For these candidates only astrometric follow-up can definitively establish their nature.

Question: Isn't it a concern that RV and direct imaging probe different ages of target stars, and so planets?

AnsweR: That's correct, RV surveys are largely targeting old, quiet stars, though some recent surveys have begun to search for planets around younger targets. As GPI and SPHERE find planets in the overlap region between direct imaging and RV we can begin to examine if orbital evolution between $\sim 10-100$ Myr and several Gyr is a significant factor in planet populations, if the distributions of planets in the same region of parameter space found by the different techniques is significantly different for different ages. 